\title{
Electromagnetic Interference Prediction in an In-House Power-Line Network
}

\author{
Margarita Samsó*, Miquel Ribó*, Albert-Miquel Sánchez*, Joan Ramon Regué*, Marc Aragón ${ }^{\dagger}$, Ferran Silva ${ }^{\dagger}$ \\ *Grup de Recerca en Electromagnetisme i Comunicacions (GRECO) \\ La Salle, Universitat Ramon Llull (URL) \\ Quatre Camins 2, Barcelona 08022, Spain \\ Email: albertm@salleurl.edu \\ $\dagger$ Electromagnetic Compatibility Group (GCEM) \\ Universitat Politècnica de Catalunya (UPC) \\ Jordi Girona 1-3, Barcelona 08034, Spain \\ Email: marc.aragon@upc.edu
}

\begin{abstract}
In this paper a new methodology for the prediction of the conducted-emission propagation in an in-house powerline network is presented. This methodology is based on the modal $S$-parameter characterization of the devices present in the power-line network, as well as on the modal modeling of branchline connections. The modal $S$ parameters relate common- and differential-mode waves, and give more information regarding interference propagation. With this methodology, common- and differential-mode attenuations, and modal conversion between common and differential modes can be accurately predicted, which is of great interest for power-line communication development and conducted-emission mitigation. This methodology is tested by accurately predicting the interference levels up to 100 $\mathrm{MHz}$ of an in-house power-line network composed by thermalmagnetic circuit breakers, residual-current circuit breakers, single-phase wires and light bulbs.
\end{abstract}

\section{INTRODUCTION}

In-house power-line networks (PLN) were originally designed to propagate the differential $50-\mathrm{Hz}$ power-supply signal. However, communication signals generated by powerline communication (PLC) modems or conducted interference emitted by electric and electronic devices are usually added to the mains signal. Therefore, the PLN can be considered a wide-band channel that expands to several tens of $\mathrm{MHz}$, and its proper characterization is of great interest to predict the signal propagation, enhance the PLC link, and improve the conducted-emission (CE) mitigation.

Several proposals to characterize the PLN can be found in the literature. In [1], a multi-path transfer function to model the PLN is presented. Its model parameters can be obtained from measurement techniques [1]-[3] or theoretical derivation [4]. Transmission-line theory has also been used to model the PLN conductors [5]-[8]. An interesting point of view is presented in [9]-[11], where modal analysis is used to predict the commonmode (CM) and differential-mode (DM) signal propagation.

In this paper, the modal analysis is exploited to analyze not only the propagation of the CM, DM and the modal conversion between CM and DM (produced by asymmetries in complex PLN structures), but also the modal behavior of power devices and terminal loads. Power devices such as thermal-magnetic circuit breakers (TMCBs), residual-current circuit breakers (RCCBs), wires or electric devices, are completely and rigorously modeled using their modal $S$ parameters, which relates CM and DM waves [12], [13]. The equivalent modal circuit of parallel branch-line connections has also been derived to implement and simulate a synthetic in-house PLN. Simulations and measurements show very good agreement below 100 MHz.

In order to establish the foundations of the work presented in this paper, Section II.A reviews the transformation equations to obtain the modal $S$-parameter matrices of two-port and fourport networks from the measured $S$-parameter ones. In Section II. $B$, equivalent CM and DM circuits for a parallel branch-line connection are derived from the analysis of its physical voltages and currents. In Section III, the presented methodology is experimentally validated by applying the characterization methodology presented in Section II on actual PLN devices and by comparing the CE in a synthetic PLN obtained from simulation and measurements. Finally, the conclusions are presented in Section IV.

\section{Characterization of Power-Line Circuits}

In order to accurately predict the CE propagation in a PLN, a suitable characterization of the components and devices present in the circuit, such as TMCBs, RCCBs, wires, loads or branch-line connections, is needed. In this section, complete characterizations of two-port and four-port networks, as well as the behavior of parallel branch-line connections, are presented and described.

\section{A. Modal Characterization of Two-Port and Four-Port Net-} works

A full characterization of passive two-port and four-port networks can be performed through their measured circuit $S$ parameter matrix $[S]$, considering as ports those established between the line $(\mathrm{L})$ and the ground $(\mathrm{G})$ terminals, and the neutral (N) and ground terminals (Fig. 1(a) and Fig. 1(b)). However, an analysis of the CE from a modal point of view, that is, considering the $\mathrm{CM}$ and DM propagation instead, is 
more adequate to understand the propagation phenomena. In order to obtain a modal characterization, an equivalent $S$ parameter matrix that relates CM and DM waves can be used, commonly called mixed or modal $S$-parameter matrix ([S $\left.S_{M}\right]$ ) [12], [13]. The modal $S$-parameter matrix of a twoport network, as seen in Fig. 1(c), can be computed from the measured $S$ parameters of the circuit of Fig. 1(a) as [12]

$$
\left[S_{M}\right]=\frac{1}{2}\left[\begin{array}{cc}
1 & 1 \\
1 & -1
\end{array}\right][S]\left[\begin{array}{cc}
1 & 1 \\
1 & -1
\end{array}\right] .
$$

The modal $S$-parameter matrix of a four-port network, as seen in Fig. 1(d), can be computed from the measured $S$ parameters of the circuit of Fig. 1(b) as [13]

$$
\left[S_{M}\right]=\frac{1}{2}\left[\begin{array}{cccc}
1 & 1 & 0 & 0 \\
1 & -1 & 0 & 0 \\
0 & 0 & 1 & 1 \\
0 & 0 & 1 & -1
\end{array}\right][S]\left[\begin{array}{cccc}
1 & 1 & 0 & 0 \\
1 & -1 & 0 & 0 \\
0 & 0 & 1 & 1 \\
0 & 0 & 1 & -1
\end{array}\right]
$$

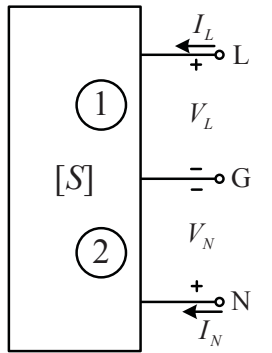

(a)

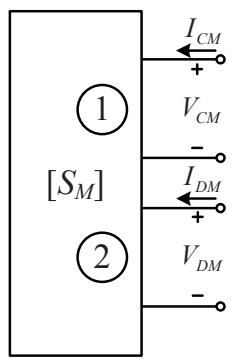

(c)

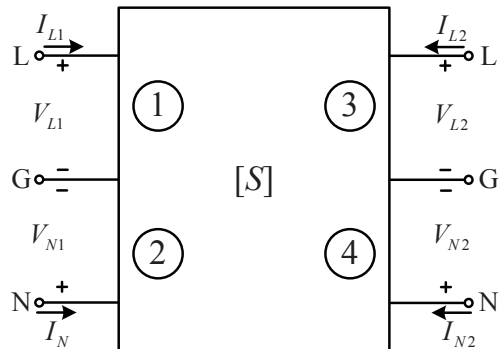

(b)

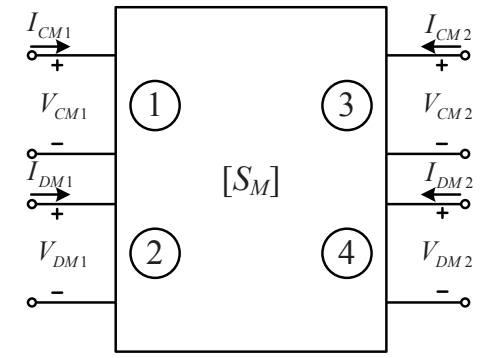

(d)
Fig. 1. Port definition for two- and four-port devices. (a) Circuit port definition for a two-port device. (b) Circuit port definition for a fourport device. (c) Equivalent modal port definition for a two-port device. (d) Equivalent modal port definition for a four-port device.

\section{B. Modal Characterization of Parallel Branch-Line Connec- tions}

Branch-line connections are commonly found in the PLN. They are needed to supply the power to all the electric devices connected to the power grid (Fig. 2). As before, an equivalent modal model of this connection is preferable in order to analyze the propagation of the CM and the DM and to interconnect the equivalent modal models of the two-port and four-port models presented above.

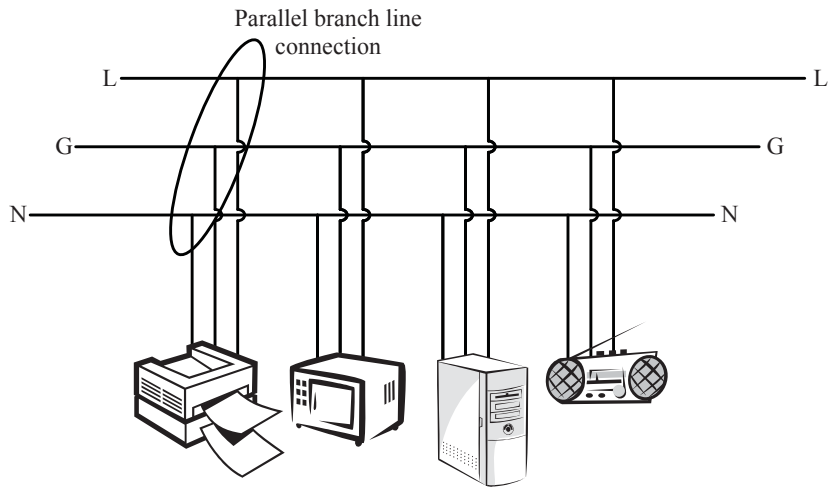

Fig. 2. Parallel branch-line connections in an in-house power grid.

The circuit and modal currents and voltages involved in a six-port parallel branch-line connection are drawn in Fig. 3. $I_{L X}, I_{N X}, V_{L X}$ and $V_{N X}(X=1,2,3)$ are the circuit currents and voltages in line and neutral ports respectively. $I_{C M X}, I_{D M X}$, $V_{C M X}$ and $V_{D M X}(X=1,2,3)$ are the modal quantities instead. The modal model of this circuit can be derived using the relationship between the circuit and the modal currents and voltages as [12], [13]

$$
\begin{aligned}
& V_{L X}=V_{C M X}+\frac{V_{D M X}}{2}, I_{L X}=\frac{I_{C M X}}{2}+I_{D M X} \\
& V_{N X}=V_{C M X}-\frac{V_{D M X}}{2}, I_{N X}=\frac{I_{C M X}}{2}-I_{D M X} .
\end{aligned}
$$

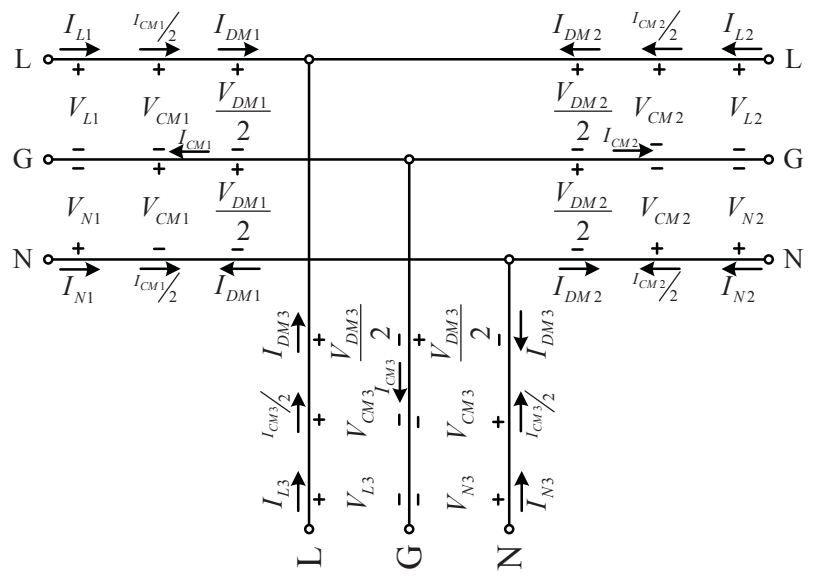

Fig. 3. Voltage and current definitions for the circuit and modal excitations in a branch line.

By analyzing the circuit of Fig. 3, the relation between the circuit voltages is

$$
\begin{aligned}
& V_{L 1}=V_{L 2}=V_{L 3} \\
& V_{N 1}=V_{N 2}=V_{N 3} .
\end{aligned}
$$

Substituting (3) into (4), the relation between the modal voltages is obtained as 


$$
\begin{aligned}
& V_{C M 1}=V_{C M 2}=V_{C M 3} \\
& V_{D M 1}=V_{D M 2}=V_{D M 3} .
\end{aligned}
$$

A similar analysis can be done considering the circuit currents in the circuit of Fig. 3 as

$$
\begin{aligned}
& I_{L 1}+I_{L 2}+I_{L 3}=0 \\
& I_{N 1}+I_{N 2}+I_{N 3}=0 .
\end{aligned}
$$

Again by substituting (3) into (6) and solving for the modal magnitudes, the relation between the modal currents are obtained as

$$
\begin{aligned}
& I_{C M 1}+I_{C M 2}+I_{C M 3}=0 \\
& I_{D M 1}+I_{D M 2}+I_{D M 3}=0 .
\end{aligned}
$$

It can be seen from (5) and (7) that there is not modal conversion between the CM and the DM. Therefore, the CM and DM equivalent modal models for the circuit of Fig. 3 that satisfies (5) and (7) are shown in Fig. 4.

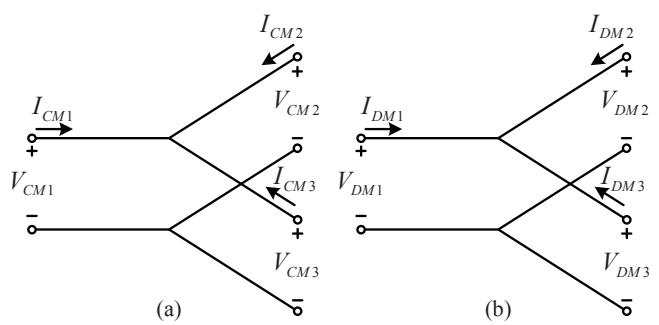

Fig. 4. CM (a) and DM (b) equivalent modal models for the circuit of Fig.

\section{EXPERIMENTAL VALIDATION}

In order to predict the interference propagation in an inhouse PLN, the modal models presented in Section II have been applied in this section to characterize several circuit components, such as TMCBs, RCCBs, single-phase wires, light bulbs and branch-line connections. In Subsection III.A, three examples of these characterizations, a RCCB, a 15-m single-phase wire and a 100-W light bulb, are presented and discussed. In Subsection III.B, the modal models are used to predict the interference propagation in a synthetic circuit that emulates an in-house PLN. To prove the robustness of the proposed methodology, measurements and simulations up to $200 \mathrm{MHz}$, well beyond the typical PLC frequency ranges, are presented.

\section{A. Component Measurements}

The $S$ parameters of a Lemag ND 62 RCCB, a 15-m singlephase wire and a 100-W light bulb have been measured. Their modal $S$-parameter matrices have been obtained using (1), in the bulb case, and (2), in the RCCB and wire cases.

Fig. 5 shows the CM attenuation $(S(3,1)$, as seen in Fig. $1)$, the DM attenuation $(S(4,2))$ and the modal conversion between CM and DM $(S(2,1)$ and $S(3,4))$ of the RCCB. It

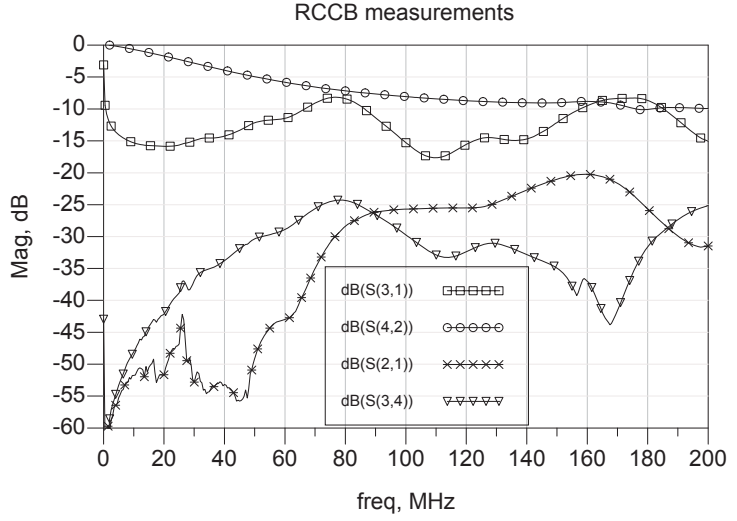

Fig. 5. Modal behavior of a Lemag ND 62 RCCB.

can be seen that both the attenuation and the modal conversion increase as frequency increases, presenting differences between 10 and $20 \mathrm{~dB}$ at $200 \mathrm{MHz}$ [14].

Fig. 6 shows the modal behavior of the 15-m single-phase wire. Again, the CM and DM attenuation and the modal conversion increase as frequency increases, achieving similar levels around $200 \mathrm{MHz}$.

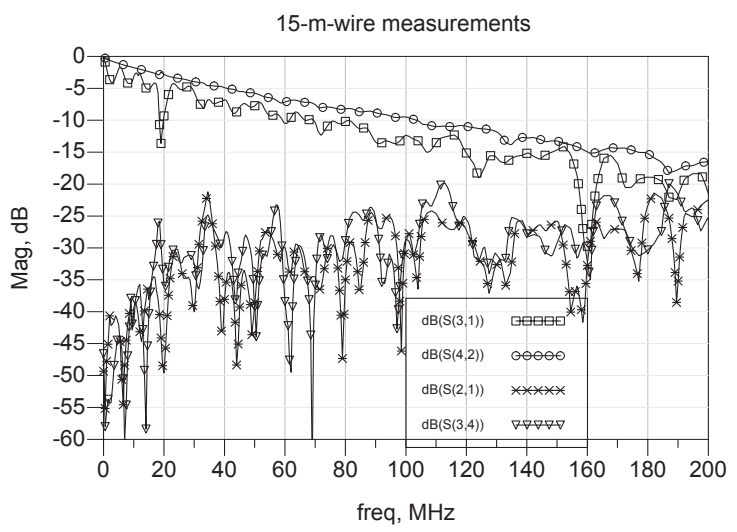

Fig. 6. Modal behavior of a 15-m single-phase wire.

Finally, Fig. 7 shows modal $S$ parameters of the 100-W light bulb. As can be seen, there is a total reflection of the $\mathrm{CM}(S(1,1))$ at all frequencies. As expected, there is some absorption of the DM $(S(2,2))$ below $60 \mathrm{MHz}$. As before, the modal conversion $(S(2,1))$ increases as frequency increases.

\section{B. In-House PLN Modal Modeling}

The modal $S$ parameters obtained in the previous subsection and the modal characterization of parallel branch-line connections presented in Subsection II. $B$ have been used to simulate the circuit shown in Fig. 8, with the switch closed allowing the signal flow. This circuit is an example of a generic structure of an in-house PLN. The modal $S$ parameters of the complete circuit have been computed with a circuit simulator using the ports P1, P2, P3 and P4 as terminal ports (Fig. 8). The same circuit has been physically implemented and its $S$ parameters 


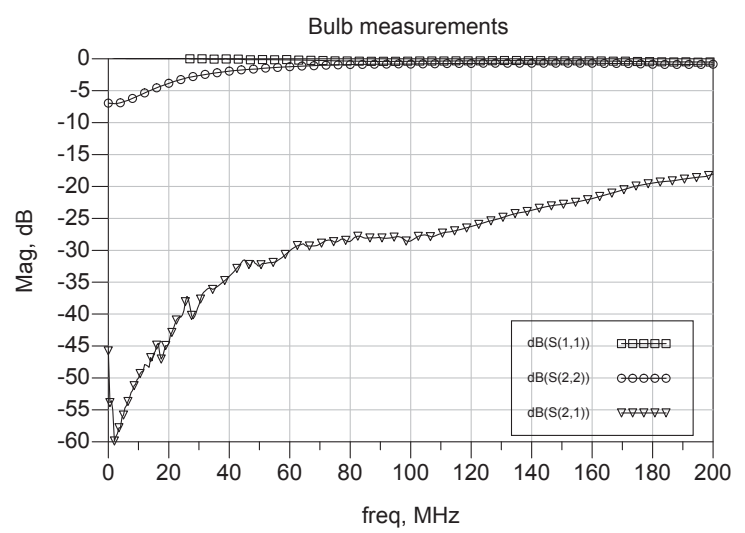

Fig. 7. Modal behavior of a 100-W bulb.

have been measured using the same terminal ports. The modal $S$-parameter matrix has been obtained using (2).

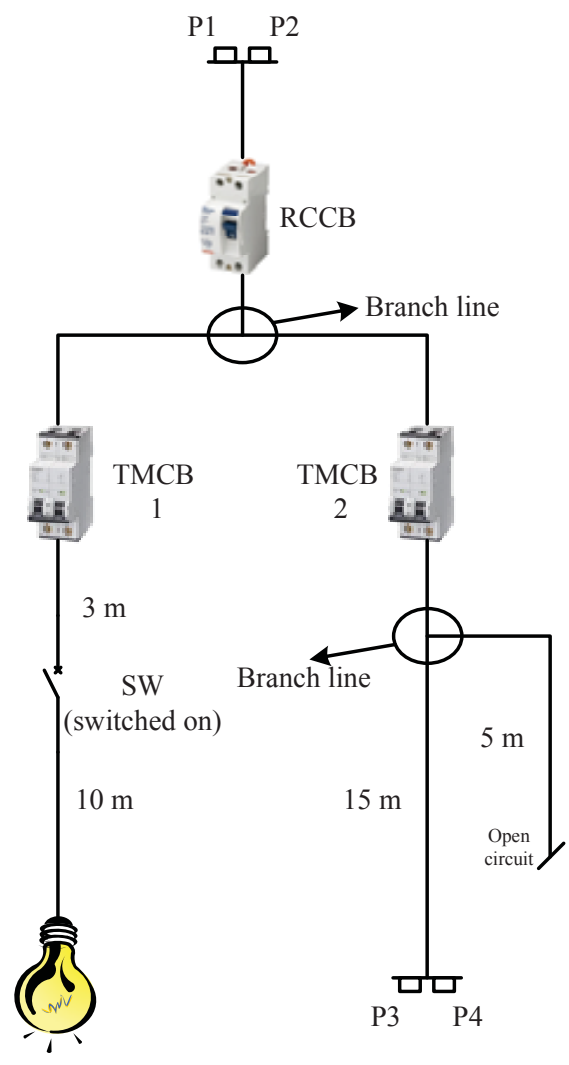

Fig. 8. Generic circuit of an in-house PLN.

Fig. 9 and Fig. 10 show the comparison between the predicted (obtained from the computed results using the circuit simulator) and the measured (obtained from the $S$-parameter measurements after applying (2)) CM and DM attenuation. As can be seen, there is a very good agreement between prediction and measurement below $100 \mathrm{MHz}$. Above $100 \mathrm{MHz}$ the general tendencies are also well predicted.

Fig. 11 and Fig. 12 show the comparison between the

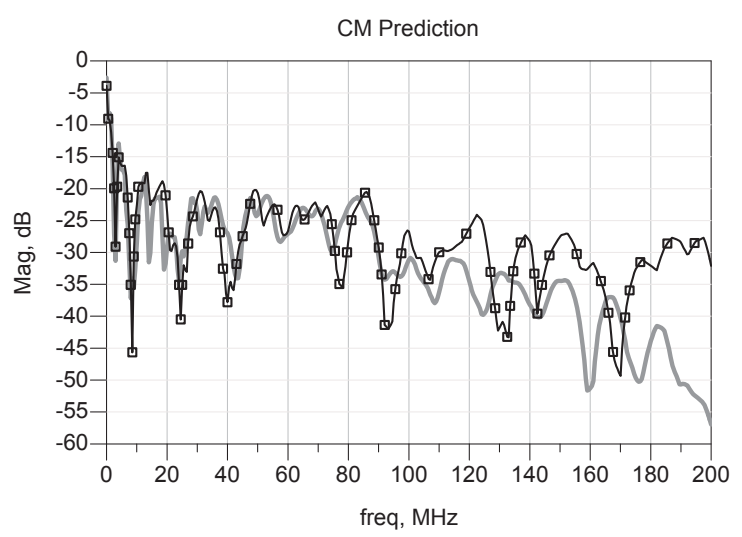

Fig. 9. Comparison between the predicted (solid line) and measured (squared line) $\mathrm{CM}$ attenuation.

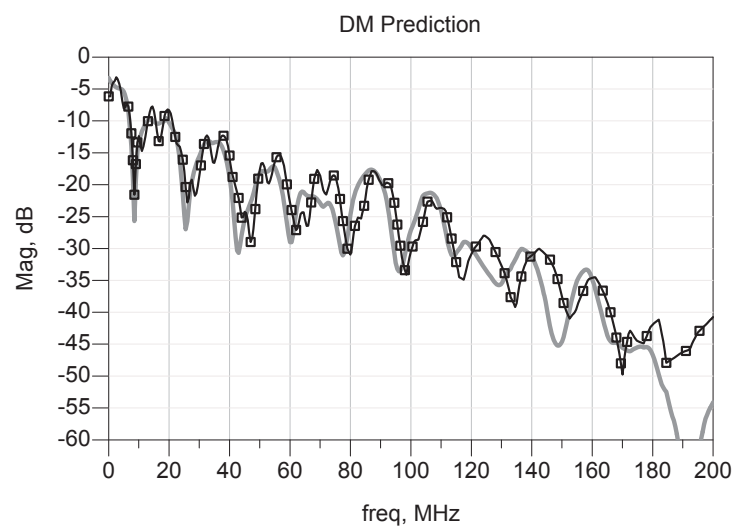

Fig. 10. Comparison between the predicted (solid line) and measured (squared line) DM attenuation.

predicted and measured modal conversion at both sides of the circuit. Again, a good agreement is obtained below $100 \mathrm{MHz}$ and the tendencies are well predicted above $100 \mathrm{MHz}$. Even in the case of very poor levels, as shown in Fig. 11 (around $-55 \mathrm{~dB}$ below $20 \mathrm{MHz}$ ), the modal behavior of the circuit is accurately predicted.

It is apparent from Fig. 9-12 that at some frequencies modal conversion is as significant as single-mode propagation to explain the presence of $\mathrm{CM}$ or DM at any given port. If the DM signal of a PLC modem is present at those frequencies, it will be mainly converted to the $\mathrm{CM}$, degrading the overall PLC performance. By predicting the PLN channel, those frequencies can be avoided by the PLC modem. Besides, power-line filters can be improved by knowing the modal behavior and input impedance of the PLN. Finally, it should be noted that using the in-house PLN modal modeling described above and a complete characterization of PLC transmitters and receivers [13], the CM and DM levels generated at any point of the network could be predicted. 


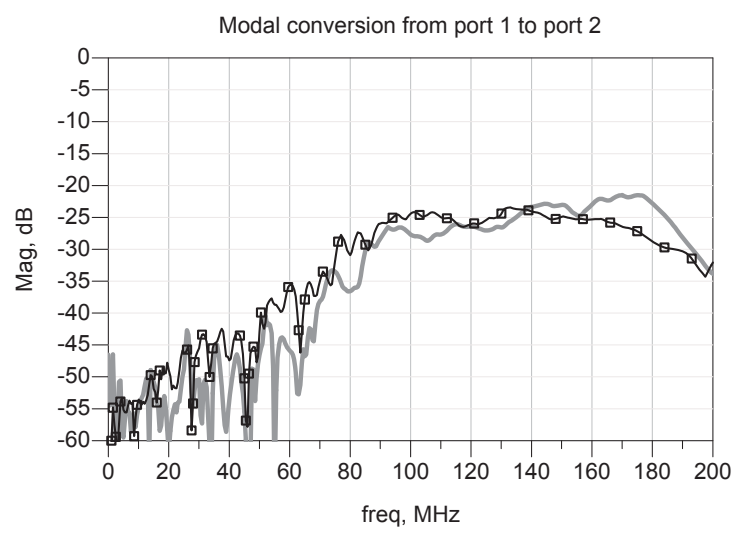

Fig. 11. Comparison between the predicted (solid line) and measured (squared line) modal conversion from port 1 to port 2 .

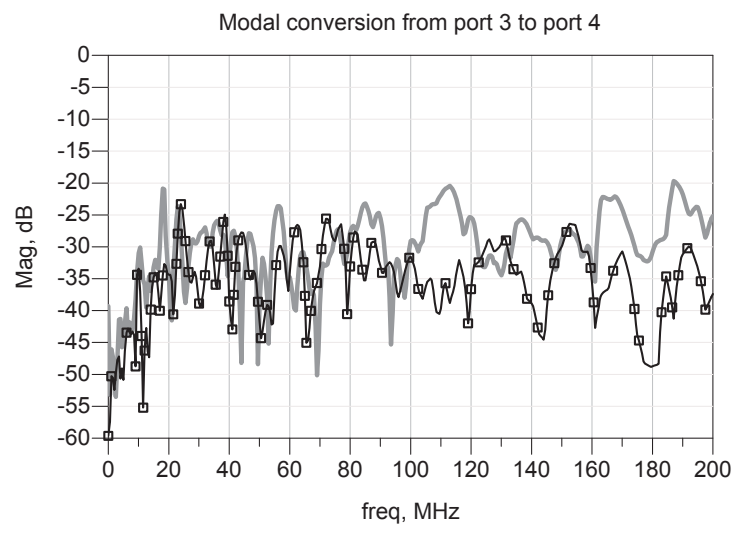

Fig. 12. Comparison between the predicted (solid line) and measured (squared line) modal conversion from port 3 to port 4 .

\section{Conclusion}

In this paper, a complete methodology to predict the modal behavior of complex power-line networks has been presented. To this end, a modal characterization methodology to model two-port and four-port networks, as well as parallel branch-line connections has been used. Two-port and four-port networks, useful to model power-line network devices such as thermalmagnetic circuit breakers, residual-current circuit breakers, single-phase wires or terminal loads, are characterized using their modal $S$ parameters, which are derived from the measured circuit ones. Parallel branch-line connections, used to distribute the power supply to electric or electronic devices, are characterized by their equivalent modal circuit. All the characterizations have been applied on actual devices and used to simulate a synthetic in-house power-line network. The same network has been physically implemented and measured. Modal simulations and measurements show a very good agreement below $100 \mathrm{MHz}$. Above $100 \mathrm{MHz}$, the simulations predict adequately the measurement tendencies. This proves that it is possible to accurately predict the conducted emissions in complex power-line networks. This is very useful to understand the modal conversion mechanisms operating in a power-line network, as well as interference and power-line communication signals propagation.

\section{REFERENCES}

[1] M. Zimmermann and K. Dostert, "A multipath model for the powerline channel", IEEE Trans. Commun., vol. 50, pp. 553-559, Apr. 2002.

[2] D. Anastasiadou and T. Antonakopoulos, "Multipath charecterization of indoor power-line networks", IEEE Trans. Power Del., vol 20, no. 1, pp. 90-99, 2005.

[3] R. Hashmat, P. Pagani, A. Zeddam and T. Chonave, "A channel model for multiple input multiple output in-home power line networks", in Proc. IEEE Int. Symp. Power Line Commun. and Its Appl., Apr. 2011, pp. 3541.

[4] L.T. Tang, P.L. So, E. Gunawan, Y.L. Guan, S. Chen, and T.T. Lie, "Characterization and modeling of in-building power lines for highspeed data transmission”, IEEE Trans. Power Del., vol. 18, pp. 69-77, Jan. 2003.

[5] T. Calliacoudas and F. Issa, "Multiconductor transmission lines and cables solver, an efficient simulation tool for PLC wetworks development," in Proc. IEEE Int. Symp. Power Line Commun. and Its Appl., Mar. 2002.

[6] H. Meng, S. Chen, Y.L. Guan, C. L. Law, P.L. So, E. Gunawan and T.T. Lie, "Modeling of transfer characteristics for the broadband power line communication channel", IEEE Trans. Power Del., vol. 19, pp. 1057-1064, Jul. 2004.

[7] I.C. Papaleonidopoulos, C.G. Karagianopoulos, N.J. Theodorou, C.N. Capsalis, "Theoretical transmission-line study of symmetrical indoor triple-pole cables for single-phase HF signalling", IEEE Trans. Power Del., vol. 20, no. 2, pp. 646-654, Apr. 2005.

[8] D. Sabolic, A. Bazant and R. Malaric, "Signal propagation modeling in power-line communication networks", IEEE Trans. Power Del., vol 20, no. 4, pp. 2429-2436, Oct 2005.

[9] T.A. Papadopoulos, Ch.G. Kaloudas and G.K. Papagiannis, "A multipath channel model for PLC systems based on nodal method and modal analysis", in Proc. IEEE Int. Symp. Power Line Commun. and Its Appl., Mar. 2007, pp. 278-283.

[10] A. Pérez, A.M. Sánchez, J.R. Regué, M. Ribó, R. Aquilué, P. RodríguezCepeda and F.J. Pajares, "Circuital and modal characterization of the power line network in the PLC band," IEEE Trans. Power Del., vol. 24, no. 3, pp. 1182-1189, Jul. 2009.

[11] G.A. Franklin, "Using modal analysis to estimate received signal levels for a power-line carrier channel on a $500-\mathrm{kV}$ transmission line", IEEE Trans. Power Del., vol. 24, no. 4, pp. 2446-2454, Oct. 2009.

[12] A.M. Sánchez, S.A. Pignari, J.R. Regué and M. Ribó, "Device Modeling for Non-Stationary Conducted Emissions Based on Frequency- and TimeDomain Measurement", IEEE Trans. Electromagn. Compat., accepted.

[13] A. Pérez, A.M. Sánchez, J.R. Regué, M. Ribó, J.P. Rodríguez-Cepeda and F.J. Pajares, "Characterization of power-line filters and electronic equipment for conducted-emission prediction", IEEE Trans. Electromagn. Compat., vol. 50, pp. 577-585, Aug. 2008.

[14] M. Ribó, J.R. Regué, F.J. Pajares, I. Gaspar, C. Vilella and J.L. Pijoan, "Propagation of PLC signals through circuit breakers", in Proc. EMC Europe Workshop, Electromagn. Compat.: Safety, Reliability and Security of Communication and Transportation Systems, Jun. 2007. 